\title{
PARP-inhibitors in a non-oncological indication as COVID-19: are we aware about its potential role as anti-thrombotic drugs? The discussion is open.
}

\author{
Ettore Capoluongo ${ }^{1}$ \\ ${ }^{1}$ University of Naples Federico II School of Medicine and Surgery
}

May 21, 2020

Capoluongo E.

PARP-inhibitors in a non-oncological indication as COVID-19: are we aware about its potential role as anti-thrombotic drugs? The discussion is open.

Department of Molecular Medicine and Medical Biotechnology

Department of Laboratory and Transfusion Medicine, Azienda Ospedaliera Universitaria Federico II, Naples

Federico II University - CEINGE, Advanced Biotechnology, Naples

email: ettoredomenico.capoluongo@unina.it

In the last three months, most research groups have shifted their focus to the fight against the COVI-2 infection (COVID-19). Many scientists worked to understand how the virus is transmitted and able to mutate or how it can be sensitive to new and old drugs. Up to now, the results obtained in this field were not always promising and, sometimes, completely inconclusive, as in the case of chloroquine (1). However, the recent discovery on benefits deriving from use of such anticoagulants for Covid-19 patients, has increased the success of treatment (2). In fact, as reported by Tang et al (2), since the disseminated intravascular coagulation and coagulopathy can contribute to patients' death, anticoagulant administration resulted as being associated with decreased mortality in severe COVID-19 pneumonia (2). Tang et al(2) also assessed that low molecular weight heparin (LMWH) regimen seems to be associated with better prognosis in severe COVID-19 patients meeting sepsis-induced coagulopathy criteria or with markedly elevated D-dimer. Along with other experimental treatments, or in combination, these findings have dramatically reduced the number of patients needing intensive cares, with a lightening of the workload of the emergency departments and a definitive improvement in the ratio of deaths to infected $(2,3)$. However, although with some concerns mainly related to the schemes of LMWH administration, using of prophylactic-doses of LMWH was recommended by the International Society on Thrombosis and Haemostasis (ISTH) for all recovered COVID-19 patients, except for those with an active haemorrhage or with a platelet count $<25 \times 10^{9} / \mathrm{L}$. (3).

While many drugs are still under investigation in clinical trials (4), after a rapid approval by the medical agencies of different countries, there are no definitive results. In this regard, very recently, a research group focused on different treatments administered to Chinese individuals showing as there is no proven regimen from conventional medicine (4). Nevertheless, most reports managed the patients with lopinavir/ritonavir, ribavirin, beta-interferon, glucocorticoid and supportive treatment with remdesivir undergoing clinical trial (4). After an extensive revision of the latest national and provincial clinical guidelines, retrospective cohort studies, and case series regarding the treatment of COVID-19 by add-on Chinese medicine, Chan et al. (4) 
concluded that, due to the paucity of strongly evidence-based treatments, the current data only suggest that Chinese medicine could be considered as an adjunctive therapeutic option in the management of COVID-19.

In this scenario, where nothing seems to be completely clear, and after reading the paper published by Berger et al (5), I would like to open the discussion on the following topic that has not been covered by the current literature.

I am referring to the fact that no available data are reported about any possible relationship between Covid2 effects and patients under chemotherapy regimen with PARP-1 inhibitors. My empirical consideration, not still supported by literature data, emerged from my experience gained in the last ten years research on the oncogenetic features of PARP-inhibition in women suffering from ovarian cancer (6). Noteworthy, patients under anti-PARP-1 regimens (5), particularly those with pancreatic, prostate, ovarian and breast cancers (most of them enrolled in clinical trials), might be more protected by the Covid-2 severe effects like the thrombotic events. I underline as for bevacizumab drug an association with venous thromboembolism has been reported in ovarian cancer patients, mostly in patients with elevated D-dimer level and high BMI before chemotherapy (5). These findings were never reported for Olaparib. Regarding PARP pathway, several studies showed as the inhibition of poly-ADP ribose polymerase-1 (PARP-1) reduced organ dysfunction in post-myocardial infarction remodelling, ischemia-reperfusion injury, diabetic retinopathy, septic shock, diabetes, and atherosclerosis, attenuating diseases associated with vascular smooth muscle and endothelial dysfunction. The latter is a specific feature of Covid-19 (3). Mathews et al. (7) demonstrated that PARP-1, when activated by oxidative and nitrosative stress, consumes cellular energy and precipitates endothelial cell death. Therefore, inhibition of PARP-1 prevented ROS- and RNS-induced HUVEC death, not only by maintaining cellular energy, but also through a novel mechanism via VEGFR2, Akt, and Bad phosphorylation. All these findings are very interesting when translated to the possible mechanisms surrounding the disseminated intravascular coagulation and coagulopathy processes leading to either patient's severe symptoms or death related to Covid-19. Noteworthy, the inflammatory process, cytokine storm, and lung injury can result in an increased risk of thrombosis for Covid-2 positive patients $(3,4)$. In this setting, use of anti-PARP drugs in non-oncological indications, although still under debate, was reported as being of benefit inflammatory disorders such as arthritis, psoriasis, colitis, asthma, diabetic complications and cardiovascular diseases (5). Moreover, although data on total incidence of thrombotic events in COVID-19 is still uncertain, we can assume that individuals with increasing age, obesity, comorbidities and cancer are at higher risk of these events. Based on the scientific evidences regarding a potential anti-inflammatory and anti-thrombosis effect of PARP-1 inhibitors (5), the scientific community should be encouraged to investigate the PARP-1-inhibitors in the treatment of Covid-19, particularly due to the mitigation effects of all pathways promoting the inflammatory and thrombotic cascades, respectively $(2,3)$.

PARP enzymes (PARP-1 and PARP-2) play a pivotal role in sustaining the genome stability. PARP inhibitors are small molecule mimetics of nicotinamide which bind to PARP's catalytic domain to inhibit poly-ADP-ribosylation (PARylation) of target proteins (5). Considering that different forms of PARP inhibitors are present now on the market (olaparib, veliparib, talazoparib, niraparib and rucaparib), there will be an easy access to these medications for using in either new clinical trials and/or in translational studies.

Finally, although a risk of genotoxicity can be also associated to PARP-1 inhibitors long term administration (5), this issues alone should not be a reason to exclude a study drug for testing in a non-oncological indication, like Covid-19 condition. As for LMWH, also in this case different schemes of administration should be evaluated in order to achieve the best benefit for patients. Therefore, I agree with Berger et al (5) who stated that there are no sufficient reasons to exclude studies based on PARP inhibitor in other non-oncological settings. For these reasons, why do not encourage both basic and translational research on PARP-1 inhibitor effects and severe forms of Covid-19?

The above considerations are far from being provocative, but they are only aimed to stimulate a thought on this specific topic. I would be grateful to the Editor if, through the publication of this paper, it would allow us to open up the debate on the subject. 


\section{References}

1. Capoluongo, E.D., Amato, F., Castaldo, G. (2020). The friendly use of chloroquine in the COVID-19 disease: a warning for the G6PD-deficient males and for the unaware carriers of pathogenic alterations of the G6PD gene. Clin Chem Lab Med . 2020 Clin Chem Lab Med, 2020 Apr 24:/j/cclm.ahead-ofprint/cclm-2020-0442/cclm-2020-0442.xml

2. Tang, N., Bai, H., Chen, X., Gong, J., Li, D., Sun, Z. (2020). Anticoagulant treatment is associated with decreased mortality in severe coronavirus disease 2019 patients with coagulopathy. J Thromb Haemost , 2020;18(5):1094-1099.

3. Thachil, J., Tang, N., Gando, S., Falanga, A., Cattaneo, M., Levi, M., Clark, C., Iba, T. (2020). ISTH interim guidance on recognition and management of coagulopathy in COVID-19. J Thromb Hemost, 18(5):1023-1026

4. Chan, K.W., Wong, V.T., Tang, S.C.W. (2020). COVID-19: An Update on the Epidemiological, Clinical, Preventive and Therapeutic Evidence and Guidelines of Integrative Chinese-Western Medicine for the Management of 2019 Novel Coronavirus Disease. Am J Chin Med , 48(3):737-762.

5. Berger, N.A., Besson, V.C., Boulares, A.H., et al .(2018). Opportunities for the repurposing of PARP inhibitors for the therapy of non-oncological diseases. Br J Pharmacol;175:192-222.

6. Capoluongo, E. (2016). BRCA to the future: towards best testing practice in the era of personalised healthcare. Eur J Hum Genet, 1:S1-2;

7. Mathews, M.T., Berk, B.C.(2008). PARP-1 inhibition prevents oxidative and nitrosative stress-induced endothelial cell death via transactivation of the VEGF receptor 2. Arterioscler Thromb Vasc Biol ,28:711-717 